\title{
Plastic deformation at high temperatures of pure and Mn-doped GaSb
}

\author{
J. L. Plaza a) and E. Diéguez \\ Departamento de Física de Materiales, Facultad de Ciencias, Universidad Autónoma de Madrid, \\ Cantoblanco, 28049, Madrid, Spain \\ M. Jiménez-Melendo and A. Domínguez-Rodríguez \\ Departamento de Física de la Materia Condensada, Universidad de Sevilla, Apartado 1065, \\ 41080 Sevilla, Spain
}

(Received 1 February 2001; accepted for publication 1 August 2001)

In this work the plastic behavior of $\mathrm{GaSb}$ and $\mathrm{Mn}$-doped $\mathrm{GaSb}$ at high temperature has been analyzed. Several experiments at different constant load and temperatures around $500{ }^{\circ} \mathrm{C}$ were carried out. The parameters used in the Haasen model have been obtained experimentally and compared with the ones obtained from simulations. (C) 2001 American Institute of Physics.

[DOI: 10.1063/1.1406970]

\section{INTRODUCTION}

The narrow-band-gap III-V semiconductors are promising materials regarding the development of high quality optical modulators and heterostructure lasers, which can operate in the midinfrared $(2-5 \mu \mathrm{m})$ region. ${ }^{1}$ Microwave and optical devices have been developed. ${ }^{2-4}$ In this sense, GaSb is one of the most important III-V semiconductor compounds because a wide range of electronic and electro-optic devices are based on this material including resonant tunneling devices, ${ }^{5}$ mid infrared lasers ${ }^{6}$ and photodiodes, ${ }^{7}$ long wavelength infrared detectors, ${ }^{8}$ and thermophotovoltaic cells. ${ }^{9}$

Mn occupies an important role as a dopant in GaSb. It offers an opportunity to study chemical trends related to the interaction between carriers and magnetic spins in a different host without the need of additional doping because of the shallow acceptor levels of $\mathrm{Mn}$ in GaSb. ${ }^{10-12}$

These features make it necessary to prepare homogeneous materials with low dislocation densities in order to satisfy the requirements of the electronic and optoelectronic industries.

It is well known that, during the growth process, thermal stresses can induce the generation and propagation of dislocations, which are directly related to the plastic deformation of the material. The quantitative knowledge of deformation phenomena gives insight about the optimal conditions under which GaSb crystals can be grown with low dislocation densities. For this purpose it is very useful to use the Haasen model ${ }^{13}$ which explains the plastic deformation in tetrahedrally coordinated semiconductors such as GaSb. This model depends on several parameters, which still are not known for several materials. The knowledge of these parameters is needed in order to carry out full growth process simulations from which the conditions for lowering the dislocation density may be obtained.

In this work, several uniaxial deformation experiments at constant load, for different stresses and temperatures, have

${ }^{a)}$ Electronic mail: jl.plaza@uam.es been conducted in order to obtain the Haasen parameters for the case of pure and Mn-doped GaSb.

\section{THEORY}

The Haasen model is the most successful model regarding the plastic deformation at high temperature in tetrahedrally coordinated semiconductors. ${ }^{13}$ This model is based on the Orowan equation, ${ }^{14}$ which relates the macroscopic deformation to the microscopic processes associated with the dislocation movement and generation.

The Haasen model is represented mathematically by the following equations:

$$
\begin{aligned}
& \frac{d \varepsilon}{d t}=b N B_{0} e^{-E / k T}(\tau-A \sqrt{N})^{m}, \\
& \frac{d N}{d t}=K N B_{0} e^{-E / k T}(\tau-A \sqrt{N})^{m+1},
\end{aligned}
$$

where $\varepsilon$ is the strain, $N$ is the dislocation density, $b$ is the Burgers vector, $\tau$ is the applied stress, $E$ is the dislocation activation energy, $K$ is a constant taking into account dislocation multiplication, $k$ is the Boltzman constant, $T$ is the temperature, $A$ is a constant which is associated with the dislocations microstructure of the material, $m$ is the stress exponent which lies in the range between 1 and 2.5 for tetrahedrally coordinated semiconductors, and $B_{0}$ is a constant related to the dislocation velocity.

\section{EXPERIMENTAL DETAILS}

In order to carry out the deformation experiments, two pure and Mn-doped GaSb ingots were grown by the Bridgman method. High purity Ga and Sb (99.9999\%) and Mn $(99.9 \%)$ were used as starting materials. These two ingots were $60 \mathrm{~mm}$ long, $12 \mathrm{~mm}$ in diameter, and $40 \mathrm{~g}$ in weight.

Two single crystals with prismatic geometry were cut from the ingots. The dimensions of the samples were 4.4 $\times 3 \times 3 \mathrm{~mm}^{3}$ for pure $\mathrm{GaSb}$ and $5.4 \times 3 \times 3 \mathrm{~mm}^{3}$ for Mn-doped GaSb. The total Mn concentration in this case was obtained by means of atomic absorption analysis and found 


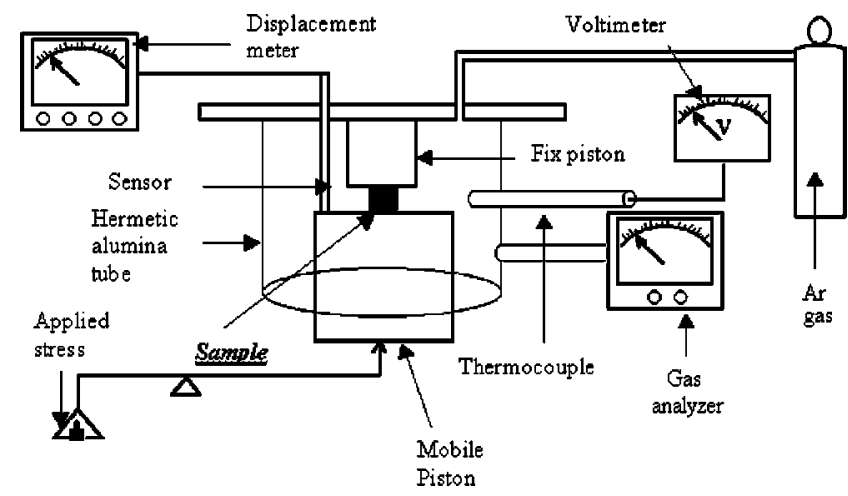

FIG. 1. Schematic of the system used for the measurements of compression at high temperature.

to be $1.8 \times 10^{19} \mathrm{~cm}^{-3}$. In order to reveal the dislocation density, the samples were mechanically polished with 5 and 1 $\mu \mathrm{m}$ alumina powder and chemically etched with $\mathrm{HNO}_{3}$ : HF: $\mathrm{CH}_{3} \mathrm{COOH}: \mathrm{H}_{2} \mathrm{O}$ (5:3:3:11). The etching process revealed a dislocation pit density of $10^{5} \mathrm{~cm}^{-3}$.

The compression faces of the samples were oriented by Laue back-reflection X-ray techniques, ${ }^{15}$ cut with a diamond saw, and polished successively with 5 and $1 \mu \mathrm{m}$ alumina powders to remove the damage generated during cutting. The orientation chosen for the compression samples was [111].

The samples were mounted in a compression apparatus which allows the execution of experiments at high temperature. A schematic of this system is shown in Fig. 1.

The experiments were carried out for compression at constant load at temperatures around $500^{\circ} \mathrm{C}\left(0.7 T_{M}\right)$ in an inert $\mathrm{Ar}$ atmosphere containing $100 \mathrm{ppM}$ of oxygen. The stress is transmitted to the sample through a mobile piston, which is placed below the sample. The sample is deformed and the mobile piston moves upwards pushing a piezoelectric transducer, which is connected to a displacement measuring apparatus. The whole system (pistons, sample, and alumina hermetic tube) is located inside a high temperature furnace, which, for sake of simplicity, is not shown in Fig. 1. The temperature is controlled by a thermocouple $\left( \pm 1^{\circ} \mathrm{C}\right)$.

\section{RESULTS AND DISCUSSION}

The samples were deformed in compression at constant load and the collected data for $\Delta l$ versus $t$ are plotted as $\ln \dot{\varepsilon}$ versus $\varepsilon$, where $\varepsilon=\left[\ln \left(/ / l_{0}\right)\right]$ with $\dot{\varepsilon}$ the strain rate. This rep-

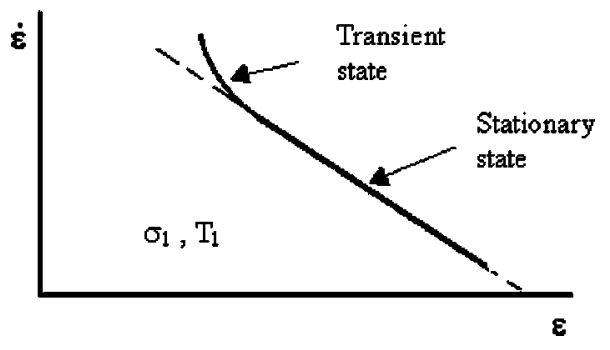

FIG. 2. Typical behavior of a strain curve at high temperature at constant stress.

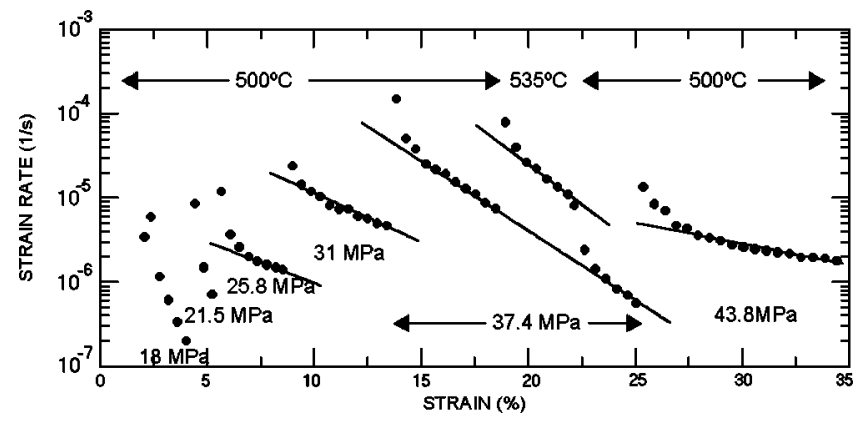

FIG. 3. Experimental results of the strain rate vs strain, from the compression experiments at high temperature for pure GaSb. The dots include the error margins.

resentation allows us to know when the stationary state is reached and if any irregularity in plastic flow occurs.

At high temperatures the strain is characterized by a stationary state where the strain rate is kept constant. Because these experiments are carried out at constant compression load, the strain rate at the stationary state decays linearly with time. This behavior is due to the fact that the sample cross section increases and therefore the stress decreases. This typical behavior is described in Fig. 2 .

We have applied the Haasen model given by Eqs. (1a) and (1b) to the stationary state in different uniaxial compression experiments at constant load for different temperatures and stresses. The special software developed by our group can simulate the evolution as a function of time, of strain, and of dislocation density by solving numerically the Eqs. (1). This makes it necessary to express the experimental points as deformation versus time instead of strain rate versus strain.

In order to solve Eqs. (1), each strain run has been considered as independent from the previous ones. In this case, two parameters are required; the initial strain and the initial density of dislocations. The former is obtained from the final

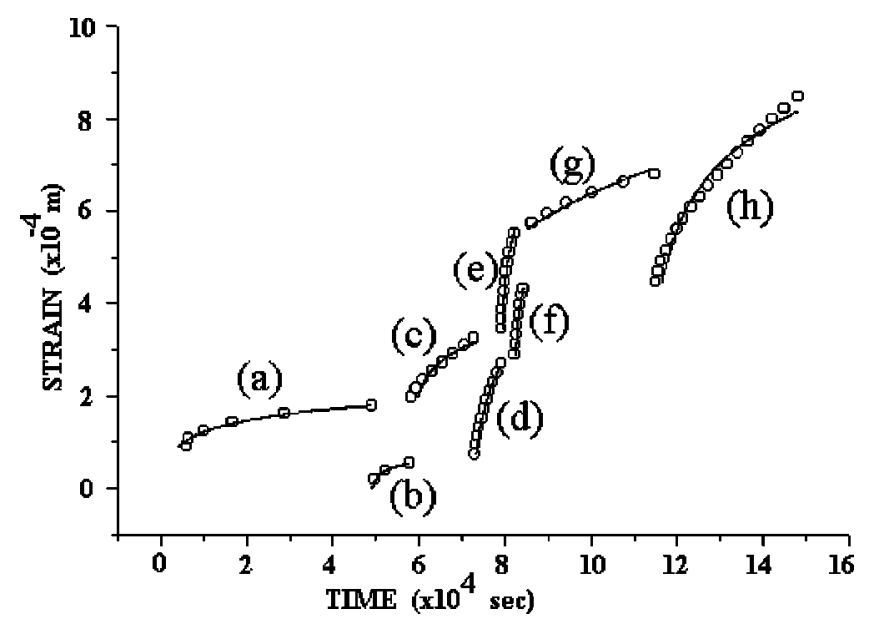

FIG. 4. Experimental (points) and simulated (continuous lines) results of the strain vs time for pure $\mathrm{GaSb}$ single crystals at different stresses and temperatures: (a) $18 \mathrm{MPa}, 500{ }^{\circ} \mathrm{C}$; (b) $21.5 \mathrm{MPa}, 500{ }^{\circ} \mathrm{C}$; (c) $25.8 \mathrm{MPa}, 500{ }^{\circ} \mathrm{C}$; (d) $31.0 \mathrm{MPa}, 500^{\circ} \mathrm{C}$; (e) $37.4 \mathrm{MPa}, 500{ }^{\circ} \mathrm{C}$; (f) $37.4 \mathrm{MPa}, 535^{\circ} \mathrm{C}$; (g) 37.4 $\mathrm{MPa}, 500{ }^{\circ} \mathrm{C}$; and (h) $43.8 \mathrm{MPa}, 500{ }^{\circ} \mathrm{C}$. The open circles include the error margins. 
TABLE I. Initial input parameters used to carry out the simulations based in the Haasen model.

\begin{tabular}{ll}
\hline \hline Burgers vector & $4.3 \times 10^{-10} \mathrm{~m}$ \\
Hardening constant $A$ & $3.1 \mathrm{~N} / \mathrm{m}$ \\
Stress exponent $m$ & 2.2 \\
Parameter $B_{0}$ & $2.5 \times 10^{-10} \mathrm{seg}^{3.2} \mathrm{~m}^{-1.1} / \mathrm{K} \mathrm{g}^{2.2}$ \\
Activation energy $E$ & $1.6 \mathrm{eV}$ \\
Initial deformation & 0 \\
Initial dislocation density $N_{0}$ & $1 \times 10^{9} \mathrm{~m}^{-2}$ \\
\hline
\end{tabular}

strain reached in the previous routine, which can be experimentally measured. Because the latter is difficult to know from the experiments, it is obtained from the simulations given by the final value presented by the evolution of the dislocation density with time in the previous run. Although this value is difficult to compare with the real ones, it is taken to be satisfactory because of the reasonable results obtained, as it will be shown. However, the initial dislocation density was measured previous to the start of the compression experiments. We found $10^{5} \mathrm{~cm}^{-2}$ for both samples.

Different compression runs have been carried out for stresses ranging from 18 to $43.8 \mathrm{MPa}$ and for temperatures between 500 and $535{ }^{\circ} \mathrm{C}$ on pure and $\mathrm{Mn}$-doped $\mathrm{GaSb}$ samples. During each routine both the temperature and the applied stress were kept constant.

\section{A. Pure GaSb}

In this case, eight different consecutive compression runs were carried out. The samples were deformed until acceleration in the strain rate was observed as shown in Fig. 3, indicating that another mechanism starts to be activated. The straight lines indicate the stationary state for different routines. It is clear that only a few points depart from the stationary state. It can also be seen that the slope progressively increases in the stationary state with increasing stress. This could be due to the hardening of the material as a consequence of the increasing dislocation density. ${ }^{13}$ This behavior is taken into account by the term $A \sqrt{ } N$ in the Haasen model [Eqs. (1)] and known as a backstress term. ${ }^{13}$ Another indication of this hardenning effect is that the slope of the curves at the stationary state is around 15 higher than the stress exponent of the Haasen model (around 2.5 as it will be shown later). Normally this slope coincides with the stress exponent. This discrepancy is a clear indication of the hardening effect due to the accumulation of dislocations.

These experimental results have been plotted as strain vs time and simulated by using our software based on the Haasen model. These results and the numerical simulations are shown in Fig. 4. Good agreement is obtained between the experimental points and the simulations. The largest departures from the simulations are coming from those experimen-

TABLE II. Haasen parameters for pure GaSb.

\begin{tabular}{cccc}
\hline \hline $\begin{array}{c}\text { Multiplication } \\
\text { constant, } K \\
(\mathrm{~m} / \mathrm{N})\end{array}$ & $\begin{array}{c}\text { Stress exponent, } \\
m\end{array}$ & $\begin{array}{c}\text { Activation energy } \\
E(\mathrm{eV})\end{array}$ & $\begin{array}{c}\text { Hardening constant } \\
A(\mathrm{~N} / \mathrm{m})\end{array}$ \\
\hline $3.8 \times 10^{-5}$ & 2.70 & 2.8 & 8.5 \\
\hline \hline
\end{tabular}

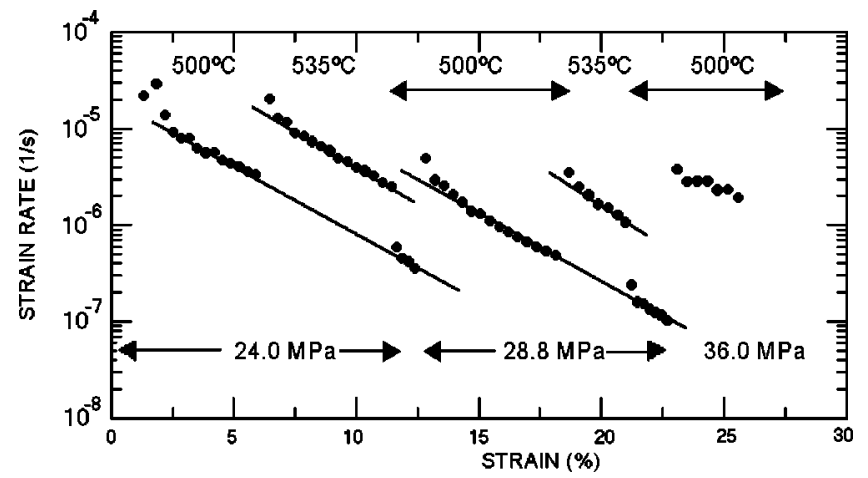

FIG. 5. Experimental results of the strain rate vs strain, from the compression experiments at high temperature for $\mathrm{Mn}$-doped $\mathrm{GaSb}$. The experimental points include the error margins.

tal points associated with the transient state. The basic input parameters used for these simulations are given in Table I. These initial trial values for the known parameters have been taken from the literature. ${ }^{15}$

The Haasen parameters for pure GaSb, obtained from the fit of these simulations to the experimental results, are given in Table II. We notice that the values obtained in this work are in good agreement with those reported previously and shown in Table I. ${ }^{15}$ To our knowledge, the parameter $K$, that is related to the dislocation multiplication, was unknown. ${ }^{16}$ It is interesting that the value for $K$ presented here for $\mathrm{GaSb}$ and obtained from the best fit is of the same order of magnitude as the one related to GaAs, being 8.8 $\times 10^{-5} \mathrm{~m} / \mathrm{N}^{15,16}$

\section{B. GaSb:Mn}

The experimental results in terms of strain rate vs strain for Mn-doped GaSb are shown in Fig. 5. In this case, the slope of the straight line, which represents the stationary state, remains almost constant for the different runs. It must be pointed out that the maximum stress is lower than in the

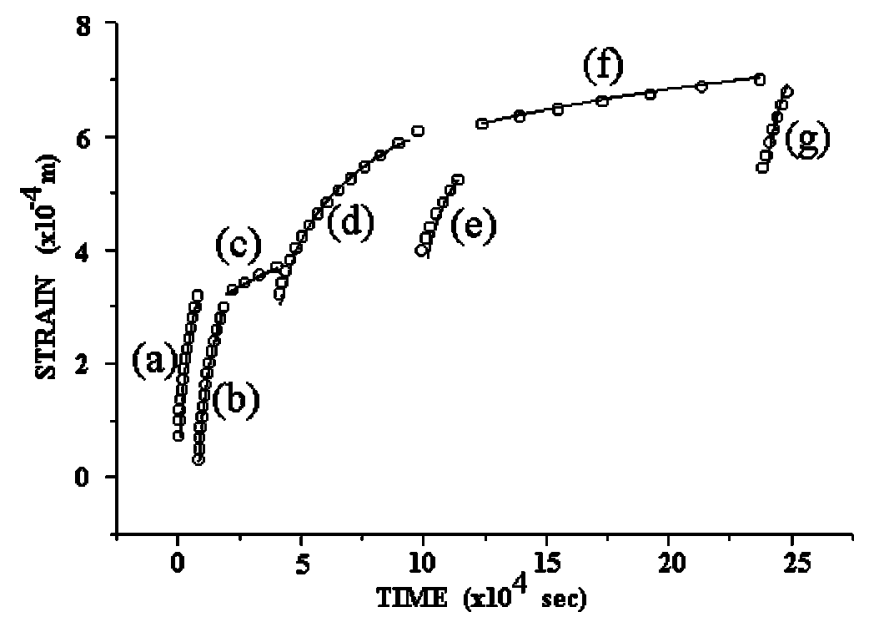

FIG. 6. Experimental (points) and simulated (continuous lines) results of the strain vs time for Mn-doped GaSb single crystals at different stresses and temperatures: (a) $24.0 \mathrm{MPa}, 500{ }^{\circ} \mathrm{C}$; (b) $24.0 \mathrm{MPa}, 535^{\circ} \mathrm{C}$; (c) $24.0 \mathrm{MPa}$, $500{ }^{\circ} \mathrm{C}$; (d) $28.8 \mathrm{MPa}, 500{ }^{\circ} \mathrm{C}$; (e) $28.8 \mathrm{MPa}, 535^{\circ} \mathrm{C}$; (f) $28.8 \mathrm{MPa}, 500{ }^{\circ} \mathrm{C}$; and $(\mathrm{g}) 36.0 \mathrm{MPa}, 500^{\circ} \mathrm{C}$. The experimental points include the error margins. The open circles include the error margins. 
TABLE III. Haasen parameters for Mn-doped GaSb.

\begin{tabular}{cccc}
\hline \hline $\begin{array}{c}\text { Multiplication } \\
\begin{array}{c}\text { constant, } K \\
(\mathrm{~m} / \mathrm{N})\end{array}\end{array}$ & $\begin{array}{c}\text { Stress exponent, } \\
m\end{array}$ & $\begin{array}{c}\text { Activation energy } \\
E(\mathrm{eV})\end{array}$ & $\begin{array}{c}\text { Hardening constant } \\
\text { A }(\mathrm{N} / \mathrm{m})\end{array}$ \\
\hline $6.5 \times 10^{-5}$ & 2.6 & 2.6 & 5.9 \\
\hline \hline
\end{tabular}

case for pure GaSb (43.8 MPa). This could be the reason why no variations in the slope at the stationary state were observed in the case of Mn-doped GaSb.

The experimental data have been replotted as strain vs time in order to establish the comparison with the theoretical results obtained from the Haasen model. These simulations and the experimental results are shown in Fig. 6. It can be observed that there exists good agreement between the experimental and the theoretical results. The parameters appearing in the Haasen model equations [Eqs. (1)], shown in Table III, were obtained from these results.

Comparing Tables I and III we notice that all the values are slightly lower in the case of GaSb:Mn than for pure GaSb. However, the differences are small. A lower dislocation activation energy could indicate that Mn slightly enhances the dislocation generation and propagation, which corresponds to a lower energy for their movement across the crystalline lattice than in the case of pure GaSb. It is interesting to note that, at the doping level used in this work, the plastic properties are more sensitive than other GaSb mechanical properties like Young's modulus and hardness, which remain unmodified. ${ }^{17}$

\section{CONCLUSIONS}

In this work uniaxial compression experiments at constant load and high temperature have been carried out with pure and Mn-doped GaSb.

The simulation of these experiments by means of the Haasen model and the comparison with the experiments pro- vided the values of the Haasen parameters. These parameters will be useful for the simulation of generation and motion of dislocations induced by thermal stresses during the real growth process.

The good agreement between the experiments and the theory validate the Haasen model for the proper description of plastic deformation in GaSb.

\section{ACKNOWLEDGMENT}

This work has been supported by CICYT under Project No. ESP-98 1340.

${ }^{1}$ P. P. Paskov, J. Appl. Phys. 81, 1890 (1997).

${ }^{2}$ A. Simon, H. L. Hartnagel, J. Schikora, V. Buschmann, M. Rodewald, H. Fuess, S. Fascko, C. Koerdt, and H. Kurz, J. Appl. Phys. 83, 7716 (1998).

${ }^{3}$ E. R. Brown, J. R. Söderström, C. D. Parker, L. J. Mahoney, K. M. Molvar, and T. C. McGill, Appl. Phys. Lett. 58, 2291 (1991).

${ }^{4}$ C. L. Felix, J. R. Meyer, I. Vurgaftman, C. H. Lin, S. J. Murray, D. Zhang, and S. S. Pei, IEEE Photonics Technol. Lett. 9, 734 (1997).

${ }^{5}$ H. Kitabayashi, T. Waho, and M. Yamamoto, Appl. Phys. Lett. 71, 512 (1997).

${ }^{6}$ D. H. Chow, R. H. Miles, T. C. Hasenberg, A. R. Kost, Y. H. Zhang, H. L. Dunlap, and L. West, Appl. Phys. Lett. 67, 3700 (1995).

${ }^{7}$ J. L. Johnson, L. A. Samoska, A. C. Gossard, J. I. Merz, M. D. Jack, G. R. Chapman, B. A. Baumgratz, K. Kosai, and S. M. Johnson, J. Appl. Phys. 80, 1116 (1996).

${ }^{8}$ H. Mohseni, E. Michel, J. Sandoen, M. Razeghi, W. Mitchel, and G. Brown, Appl. Phys. Lett. 71, 1403 (1997).

${ }^{9}$ R. Beckert and K. Bücher, Proceedings of the Fourteenth European Photovoltaic Solar Energy Conference, Barcelona, Spain, 1997 (Stephens \& Associates, UK, 1998), p. 1710.

${ }^{10}$ V. Sestáková, P. Hubik, B. Stépánek, and J. Kristofik, J. Cryst. Growth 132, 345 (1993).

${ }^{11}$ L. P. Krukovskaya, I. F. Mironov, and A. N. Titkov, Fiz. Tekh. Poluprovodn. (S.-Peterburg) 12, 689 (1978).

${ }^{12}$ F. Matsukura, E. Abe, and H. Ohno, J. Appl. Phys. 87, 6442 (2000).

${ }^{13}$ H. Alexander and P. Haasen, Solid State Phys. 22, 27 (1968).

${ }^{14}$ A. Seeger, Handbook der Physik (Springer, Berlin, 1955), Vol. II 1.

${ }^{15}$ M. Omri, J. P. Michel, and A. George, Philos. Mag. A 62, 203 (1990).

${ }^{16}$ P. Boiton, Ph.D. thesis, Université de Montpellier, 1996, p. 222.

${ }^{17}$ J. L. Plaza and E. Diéguez, Phys. Status Solidi A 174, 361 (1999). 